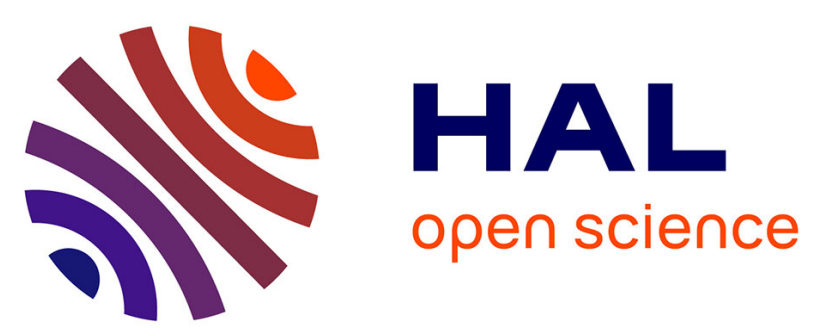

\title{
Residual-Impurity Effects in Directional Solidification: Long-Lasting Recoil of the Front and Nucleation-Growth of Gas Bubbles
}

Silvère Akamatsu, Gabriel Faivre

\section{- To cite this version:}

Silvère Akamatsu, Gabriel Faivre. Residual-Impurity Effects in Directional Solidification: LongLasting Recoil of the Front and Nucleation-Growth of Gas Bubbles. Journal de Physique I, 1996, 6 (4), pp.503-527. 10.1051/jp1:1996227 . jpa-00247200

\section{HAL Id: jpa-00247200 https://hal.science/jpa-00247200}

Submitted on 1 Jan 1996

HAL is a multi-disciplinary open access archive for the deposit and dissemination of scientific research documents, whether they are published or not. The documents may come from teaching and research institutions in France or abroad, or from public or private research centers.
L'archive ouverte pluridisciplinaire HAL, est destinée au dépôt et à la diffusion de documents scientifiques de niveau recherche, publiés ou non, émanant des établissements d'enseignement et de recherche français ou étrangers, des laboratoires publics ou privés. 


\title{
Residual-Impurity Effects in Directional Solidification: Long-Lasting Recoil of the Front and Nucleation-Growth of Gas Bubbles
}

\author{
Silvère Akamatsu (*) and Gabriel Faivre \\ Groupe de Physique des Solides $\left(^{* *}\right)$, Universités Denis-Diderot et Pierre- et Marie-Curie, \\ Tour 23, 2 place Jussieu, 75251 Paris Cedex 05, France
}

(Received 22 September 1995, received in final form 26 December 1995 and accepted 4 January 1996)

PACS.81.30.Fb - Solidification

PACS.81.10.Fq - Growth from melts; zone melting and refining

PACS.64.70.Dv - Solid-liquid transitions

\begin{abstract}
Directional-solidification experiments are often perturbed by the nucleation of gas bubbles and other residual-impunty effects. We present a detailed experimental study of these phenomena in the system $\mathrm{CBr}_{4}-\mathrm{C}_{2} \mathrm{Cl}_{6}$ directionally solidified in thin films. As is usual in this type of experıments, we use zone-refined and outgased products, but do not fill and seal the samples under vacuum. We study the solute-redistribution transient (initial recoll of the growth front) in samples of as-refined $\mathrm{CBr}_{4}$, in the absence of bubbles. This in situ method allows us to detect all the impurities present in our samples and to estimate their concentrations and partition coefficients. We point out several artefacts that may be caused by the dissolved residual impurities (shift of the cellular threshold velocity, modifications of the morphology of the cellular front). We then describe in detail the nucleation-growth process of gas bubbles during the course of solidification. There exists a steady growth pattern consisting of a periodic array of tubular bubbles similar to the rod-like pattern of eutectics and monotectics. We study the morphology of this state as a function of the growth velocity. We show that, at high velocity, the pattern decomposes in a series of independent local two-phased structures, the solid-vapour (SV) fingers, of width a few diffusion lengths. Between the SV fingers, the front is unperturbed.
\end{abstract}

\section{Introduction}

Among the numerous perturbing factors to which solidification processes are submitted, a particularly ubiquitous one is the formation of small gas bubbles in the liquid near the growth front, which are entrapped by the growing solid subsequently. This phenomenon is due to gaseous residual impurities, always present in significant concentration in the melt, unless both the purification and the solidification processes are carried out under vacuum. Since gases generally have a much lower solubility in solids than in liquids, the gas concentration in the liquid ahead of the growth front goes up to high values during the solidification process, and, in this region, bubbles of the vapour phase can nucleate [1].

$\left({ }^{*}\right)$ Author for correspondence (e-mall: akamatsu@gps.jussieu.fr)

$\left({ }^{* *}\right)$ CNRS URA 17

(C) Les Éditions de Physıque 1996 
To our best knowledge, most existing studies of the part played by gaseous residual impurities in the solidification of liquids are due to metallurgists. These generally focus on the final shape and the spatial distribution of the bubbles in the solid, which are the factors directly influencing the mechanical properties of the product, and do not pay much attention to the dynamic aspects of the perturbations caused by residual impurities [2,3]. Thanks, in particular, to the remarkable work by Chernov and Temkin [4], and Gegusin and Dzjuba [5], fundamentalist researchers in the field of crystal growth have recently begun to study experimentally the effects of residual impurities on the growth dynamics [6-10]. Basic experiments on solidification are generally carried out on transparent molecular materials, which can dissolve relatively large quantities of gases in their liquid state [11]. It is therefore no surprise that residualimpurity effects were found to be far from negligible in these experiments. Some authors tried, apparently with success, to prevent gas-bubble nucleation from occurring during their solidification experiments by performing them under a rarefied atmosphere $[6,7]$. This does not lessen the need of careful studies of the residual-gas effects during solidification, for the following reasons: first, it is not always desirable or possible to perform solidification under vacuum, and, second, bubble nucleation is not the only significant residual-impurity effect. as we shall show below.

In this article, we present an experimental study of the residual-impurity effects in the system $\mathrm{CBr}_{4}-\mathrm{C}_{2} \mathrm{Cl}_{6}$ directionally solidified in thin films. We recall that, in thin-film directional solidification, the transparent alloy to be solidified is confined in a thin glass container, and pulled at an imposed velocity $V$ in a fixed, uniform temperature gradient $G$. The shape of the growth front and its position in the temperature gradient, thus its temperature, are continuously observed with an optical microscope. In this study, we have submitted two types of materials to thin-film directional solidification, namely, as-refined $\mathrm{CBr}_{4}$ and a $\mathrm{CBr}_{4}{ }^{-}$ $\mathrm{C}_{2} \mathrm{Cl}_{6}$ alloy of concentration about $3 \mathrm{~mol} \% \mathrm{C}_{2} \mathrm{Cl}_{6}$ (thereafter called undoped and doped $\mathrm{CBr}_{4}$, respectively). We have limited ourselves to relatively low pulling velocities $\left(V \leq 35 \mu \mathrm{m} \mathrm{s}^{-1}\right)$. For the thermal gradient and the concentrations utilised, this corresponds to velocities lower than about $10 V_{c}$, where $V_{c}$ is the cellular-threshold velocity.

Our choice of the system $\mathrm{CBr}_{4}-\mathrm{C}_{2} \mathrm{Cl}_{6}$ is dictated by several considerations. First, we wish to complete a preceding investigation, devoted to the in situ quantitative determination of the physical parameters of $\mathrm{CBr}_{4}-\mathrm{C}_{2} \mathrm{Cl}_{6}$ relevant to solidification [10]. Second, $\mathrm{CBr}_{4}-\mathrm{C}_{2} \mathrm{Cl}_{6}$ is a particularly convenient "model system" for fundamental studies of the diffusion-controlled solidification dynamics. It presents a eutectic plateau ranging from about 9 to $18 \mathrm{~mol}_{0} \mathrm{C}_{2} \mathrm{Cl}_{6}$, and the two crystal phases limiting this plateau grow from the melt in a fully non-faceted way. Since its discovery by Jackson and Hunt in the 60's [12], $\mathrm{CBr}_{4}-\mathrm{C}_{2} \mathrm{Cl}_{6}$ has been utilised by many authors (see $[10,13,14]$ and earlier references therein). It is thus of interest to describe quantitatively the residual-gas effects in this particular system under the same conditions as those of the previous experiments. In brief, these conditions are that, while both $\mathrm{CBr}_{4}$ and $\mathrm{C}_{2} \mathrm{Cl}_{6}$ are carefully purified, the samples are filled and sealed in the ambient air - the main reason for not performing the experiments under a rarefied atmosphere being the high vapour pressure of $\mathrm{CBr}_{4}$ and $\mathrm{C}_{2} \mathrm{Cl}_{6}$ in the solid phase. Consequently, the system finally contains (at least) some minimal quantities of the arr components as residual impurities.

The term of residual-impurity effect applies to a number of different phenomena. The residual impurities may remain dissolved, or precipitate in the form of gas bubbles. In the latter case, the bubbles may be repeatedly entrapped by the solid or may continuously grow with it. We shall consider all these aspects in turn. To interpret them consistently, we shall need to know the equilibrium diagram of the materıal, i e., of the system $\mathrm{CBr}_{4}-\left(\mathrm{C}_{2} \mathrm{Cl}_{6}\right)$-impurity. This diagram cannot be determined by standard calormetric methods, but is (partly) deducible from the observations reported in this article. For the clarity of the presentation, it is preferable that, 


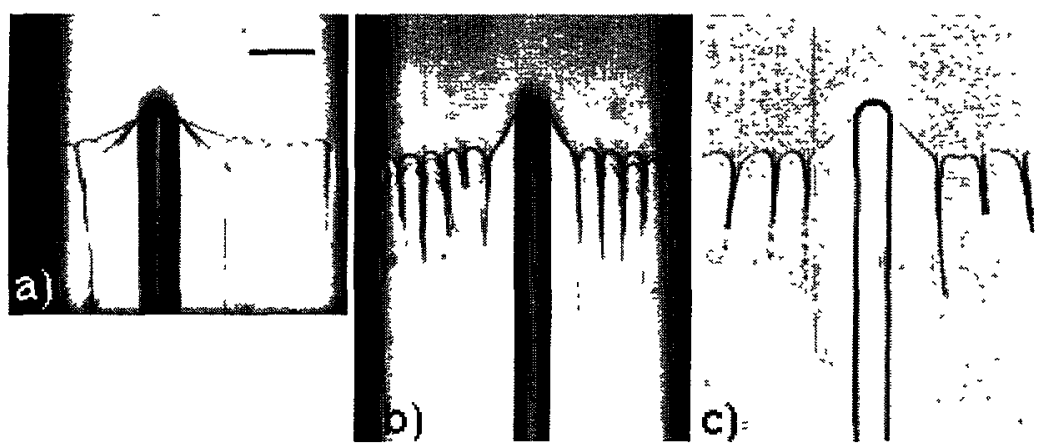

Fig. 1. - Stationary tubular gas bubbles in samples pulled at constant velocity $V$. a) undoped $\mathrm{CBr}_{4}$ in a capillary sample $\left(V=34.5 \mu \mathrm{m} \mathrm{s}^{-1}\right)$; b) $\mathrm{CBr}_{4}-\mathrm{C}_{2} \mathrm{Cl}_{6}$ alloy (doped $\left.\mathrm{CBr}_{4}\right)$ in a capillary sample $\left(V=10.7 \mu \mathrm{m} \mathrm{s}^{-1}\right)$; c) undoped $\mathrm{CBr}_{4}$ in a thin sample $\left(V=32 \mu \mathrm{m} \mathrm{s}^{-1}\right)$. The growth direction is upward. The dark regions on the left and right sides of (a) and (b) are the 1mages of the lateral wall of the capillary. The bar in a) corresponds to $100 \mu \mathrm{m}$.

anticipating on what follows, we explain now what we think of the equilibrium diagram of the system $\mathrm{CBr}_{4}$-impurity.

It will be seen below that our best samples of as-refined $\mathrm{CBr}_{4}$ (thereafter called noncontaminated undoped samples) can be considered as samples of a binary alloy $\mathrm{CBr}_{4}-\mathrm{X}$, where $\mathrm{X}$ stands for the residual impurity. Concerning the equlibrium diagram of this system, the crucial facts are:

- the impurity $\mathrm{X}$ is gaseous, i.e., it has a high affinity for the vapour phase. It can induce the nucleation of gas bubbles at the growth front. Once such a bubble has nucleated, it often assumes the stationary tubular form shown in Figure 1. This tubular pattern, as we call it, is the steady state of the system in the regime of solid-vapour coupled growth. The existence of such a steady state was previously mentioned by Gegusin and Dzuba [5], and Jamgotchian et al. [9] in various other systems;

- when no gas bubble is present along the solidification front, the residual gas manifests itself mostly through a very slow drift of the growth front towards the cold side of the directionalsolidification setup, beginning at the onset of the solidification run and generally lasting until the end of the run. Basically, this drift is nothing else than the solute-redistribution transient [15] (also called "initial recoil" of the front) due to X. It is very slow because the partition coefficient of $\mathrm{X}$ is very small. A similar long-lasting drift was noticed previously by Cladis et al. In succinonitrile [8];

- after a sufficiently long annealing, the liquid phase does not contain any gas bubble (any bubble originally present rapidly re-dissolves in the liquid). Thus the homogensed liquid phase is not saturated in $\mathrm{X}$.

The striking analogy between the stationary tubular pattern illustrated in Figure 1 and the rod-like patterns of eutectics [16] or monotectics [17] has an obvious thermodynamic meaning. Since there exists stationary triple points (in fact, lines) at which three phases are in contact, the $\mathrm{CBr}_{4}-\mathrm{X}$ equilibrium diagram presents a "plateau" at a temperature $T_{\mathrm{B}}$ (where $\mathrm{B}$ stands for bubble), corresponding to the thermodynamic equilibrium between the three following phases: the crystal at concentration $C_{S}$, the liquid at concentration $C_{\mathrm{B}}$ and the vapour at concentration $C_{\mathrm{V}}$ (Fig. 2). The other predictable features of the $\mathrm{CBr}_{4}-\mathrm{X}$ diagram are the small value of the solid-liquid partition coefficient $K^{\mathrm{X}}$, evidenced by the long characteristic time of the initial 


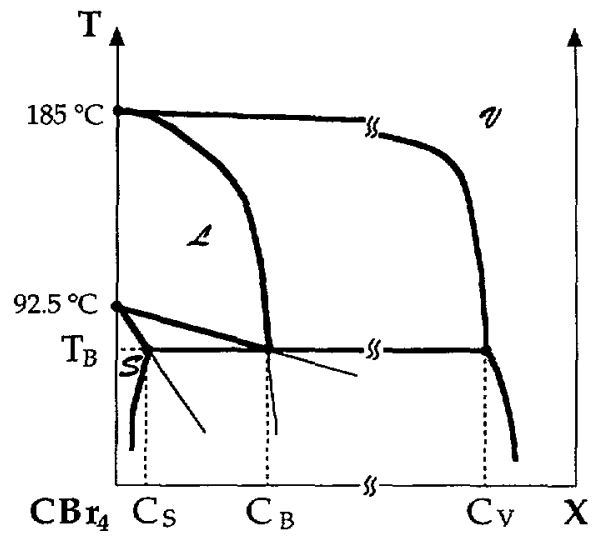

Fig. 2. - The presumed phase diagram of the $\mathrm{CBr}_{4}-\mathrm{X}$ system, where $\mathrm{X}$ stands for the chemically non-identified residual gas. S, L and V stand for solid, liquid and vapour phase. See the text for the other symbols. The concentration scale on the left-hand side is strongly expanded $\left(\mathrm{C}_{\mathrm{B}}\right.$ is of the order of $\left.10^{-3}\right)$.

transient, and the step-like shape of the liquid-vapour coexistence curve, due to the large difference between the melting points of the two pure components (the curve in Fig. 2 has actually been calculated assuming that the vapour and the liquid are ideal solutions, and taking Argon at ambient pressure for $\mathrm{X}$ ). The fact that the homogenised liquid is not supersaturated in gas implies that the average gas concentration $C_{\infty}^{\mathrm{X}}$ is positioned somewhere between $C_{\mathrm{S}}$ and $C_{\mathrm{B}}$.

Such are the essential features of the phase diagram of undoped $\mathrm{CBr}_{4}$. Those of doped $\mathrm{CBr}_{4}$ are not significantly different, since the molar fraction of the addition element $\mathrm{C}_{2} \mathrm{Cl}_{6}$ is much smaller than $1(\approx 0.03)$. It will be seen that all the experimental observations described below support - or, at least, are compatible with - the equilibrium diagram drawn in Figure 2. We shall also largely utilise the analogy between solid-vapour coupled growth and eutectic growth in order to interpret the growth dynamics of the system subsequent to the nucleation of a bubble at the front. It must however be stressed that, from the point of view of the dynamics, there are important differences between the two types of systems, namely, the large specific volume of the vapour phase compared to that of a crystal, the large surface tension of the vapour-liquid interface in comparison to the solid-liquid one, and the strong advection and convection flows induced by the bubbles in the liquid. So, although qualitatively useful, the analogy must not be presumed to hold quantitatively.

The plan of the article is as follows. In Section 2, we give details about the experimental methods. In Section 3, we consider several questions which are not central for our present purpose, but nevertheless necessary for a complete understanding of the physics of the system, namely, the wetting of the glass by the liquid, the origin of the convection flows accompanying the bubbles, and the kinetics of dissolution of the bubbles in the liquid at rest. In Section 1 , we treat the central problem of the determination of the impurity content of our materials by means of a careful analysis of the initial recoil of the front in the absence of gas bubble. We also summarily describe the effects of residual impurities on the value of the cellular-threshold velocity and the morphology of the cellular growth fronts. Section 5 is devoted to the study of the nucleation process of gas bubbles. In Section 6 , we describe the transitory process of growth of the bubbles subsequent to their nucleation, and their final steady tubular state. A brief conclusion is given in Section 7 . 


\section{Experimental Details}

The description of the thin-film directional solidification stage and the methods of purifying the products and preparing the samples can be found elsewhere [10]. We shall only discuss here the various sources of experimental uncertainty affecting the measurement of the soluteredistribution recoil.

Our standard samples are made of two parallel glass plates separated by calibrated spacers, delimiting an empty space $12 \mu \mathrm{m}$ thick, $10 \mathrm{~mm}$ wide and $70 \mathrm{~mm}$ long. Such samples provide a large aspect ratio and a strong $2 \mathrm{D}$ character to the pattern. In some cases, we prefer to use samples consisting of several rectangular captllaries (internal section $450 \times 50 \mu \mathrm{m}$, length $50 \mathrm{~mm}$ ) placed side by side. These have the advantage of being much more easy to handle, and allowing the simultaneous observation of samples of different composition. In this study, we have mostly worked with capillary samples, but we have checked that our conclusions are also valid for the glass-plate samples. The samples are carefully cleaned and kept under vacuum before being utilised (the stains often visible in the micrographs of capillary samples are on the outside face of the capillary walls).

The first source of error in the measurement of the recoil curves is the thermal mertia of the setup. The temperature distribution within the sample is established dynamically through heat diffusion along the walls of the container. It is thus different at different pulling velocities, and some time is necessary for its re-adjustment after a velocity jump, in particular, after the jump from zero to a finite value of $V$ at the onset of an experiment. A simplified theory of this "instrumental recoil", given elsewhere [18], leads to the following conclusions. Let $z$ be the axis directed from the cold to the hot side of the setup. Consider the point of the sample at some reference temperature, e.g., the equilibrium temperature $T_{0}$ of the front at $V=0$. The stationary position $z_{0}(V)$ of this point in the laboratory reference frame is a function of $V$, which decreases linearly as $V$ increases. Thus, at the onset of a solidification run, $z_{0}$ recoils (i.e., shifts towards the cold end of the setup) over a distance $\Delta z_{0}=\left|z_{0}(V)-z_{0}(0)\right|$ proportional to $V$. Provided that $z_{0}$ remains close to the edge of the hot oven (which is the case in our experiments), the temperature distribution shifts rigidly, the thermal gradient $G$ remaining constant (the dependence of $\Delta z_{0}$ on $z_{0}(0)$ is negligible). The temporal course of the instrumental recoil is exponential, with a characteristic time $\tau_{0}$ independent of $V$. For the setting corresponding to the experiments described below, we measured $G \approx 90 \mathrm{~K} \mathrm{~cm}^{-1}$, $\Delta z_{0} / V \approx 5 \mathrm{~s}$ (for capillary samples) and $\tau_{0} \approx 12 \mathrm{~s}$.

The experimentally measured recoil curves are thus a combination of the instrumental and the solute-redistribution recoil. However, the characteristic time of the latter is much longer than that of the former, as will be seen below. It is therefore unnecessary to carry out an exact de-convolution of the two components. To deduce the (purely) solutal transient from the measured recoil curve, it suffices to take the origin of time about $2 \tau_{0}$ after the onset of the pulling, and subtract the calibrated value of $\Delta z_{0}$ from the measured displacement of the front. The initial-recoil curves shown below have been corrected according to this simplified procedure.

Experimental errors are also caused by the fact that the translation of the sample is not perfectly regular. These irregularities do not stem from the motor itself, but from the micrometric screw and the sliding channel imposing a translation motion to the sample. We recorded the motion of marks made on the glass plates at constant rotation speed of the motor, and noticed two types of irregularities: erratic, small jerks of amplitude a few microns and duration much less than a second, probably due to some stick-slip in the sliding channel; a slow, periodic modulation of the translation velocity of amplitude corresponding to about $4 \%$ of the average velocity and period corresponding to a $1 \mathrm{~mm}$ translation, due to a slight misalignment of the 
micrometric screw. Both the small jerks (not felt by the system, because of the thermal inertia of the setup) and the slow modulations appear on the recorded curves, but have a short period compared to the duration time of an experimental run, and therefore only cause negligible errors in the measurements.

The most serious source of error is the possible residual inhomogeneity of the liquid at the moment of the onset of a solidification run. Let us explain this point in detail. The pre-heated capillary samples are filled simply by dipping one of their extremities into the molten alloy. This is done in the ambient air in such a way that the contact with the air lasts only a few seconds. The cooling of the capillaries to room temperature starts very rapidly. The solidification of the alloy into the high-temperature cubic crystal phase of $\mathrm{CBr}_{4}$ takes less than one second. The macro-segregation connected with the solidification is thus confined to a very small volume of the sample. At this stage, the material contains no gas bubble. Gas bubbles appear later during the cooling, when $\mathrm{CBr}_{4}$ transforms from the cubic phase to the much denser monoclinic phase [19]. This transition occurs at about $47^{\circ} \mathrm{C}$, and proceeds rather slowly from numerous nucleation centres, a few hundred microns apart from each other. It is accompanied by the nucleation of micron-size gas bubbles along the transformation front. Larger bubbles, and, occasionally, cracks also appear between the film and the glass-plates in the rear of the front.

When the sample is introduced into the solidification setup, the material on the hot side reverts first to the cubic, and then to the liquid phase. Both transformations occur very rapidly through the propagation of fronts starting from the hot extremity of the sample. respectively, and stopping at the liquid-cubic and cubic-monoclinic equilibrium temperatures. Upon this transformation, the gas bubbles present in the monoclinic phase pass into the liquid and the cubic phases. This is the origin of the bubbles present in the liquid at the beginning of the homogenisation annealing. This annealing simply consists of letting the sample immobile in the directional-solidification setup for about 1 hour. During this period of time, the bubbles initially present in the liquid generally entirely re-dissolve in the liquid (see Sect. 3.3).

We utilised a different sample for each experiment, and only a small (generally about $10 \mathrm{~mm}$ long), central portion of that sample. The reproducibility of the measured recoil curves in the non-contaminated samples convinced us that the composition inhomogeneities in the utilised part of the samples are small, as could be expected. The typical value of the diffusion coefficients in non-polymeric liquids near the melting temperature of the crystal is $5 \times 10^{-10} \mathrm{~m}^{2} \mathrm{~s}^{-1}[20]$. The diffusion distance of all species during the annealing is thus of the order of $1 \mathrm{~mm}$. Concentration fluctuations are thus damped out by diffusion over distances much longer than the average distance between the gas bubbles intially launched into the liquid.

\section{Miscellaneous Issues}

3.1. Partial Wetting of the Glass Plates by the Liquid. - The values of contact angles between the interfaces at the triple points of tubular bubbles (e.g., in Fig. 1) clearly show that, in agreement with a general rule, the surface tensions of the solid-vapour $\left(\gamma_{\mathrm{sv}}\right)$ and liquid-vapour $\left(\gamma_{\mathrm{v}}\right)$ interfaces are much larger than that of the solid-liquid interface $\left(\gamma_{s l}\right)$. That the liquid totally wets the solid $\left(\gamma_{s v}>\gamma_{l v}+\gamma_{s l}\right)$ shows up, for instance, in the fact that the bubbles crossing the solid-liquid interface at rest do not remain in contact with the solid (e.g., in Fig. 3 below).

On the other hand, liquid $\mathrm{CBr}_{4}$ wets the glass only partly. Consequently, bubbles (including tubular bubbles) are always attached to one of the glass plates enclosing the liquid. Under the optical microscope, the meniscus formed by the liquid-vapour interfaces appears as a dark line of width approximately equal to half the film thickness (compare Figs. 1a and 1c), indicating 

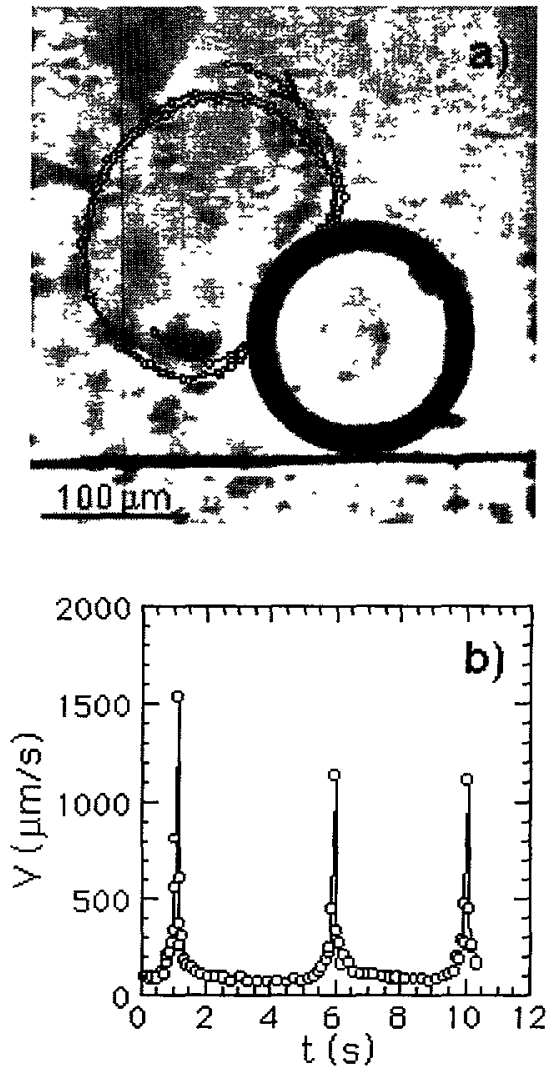

Fig. 3. - a) Trajectory of a dust particle floating in the liquid near a gas bubble in a sample of undoped $\mathrm{CBr}_{4}$ at rest; b) velocity of the dust particle versus time.

that the contact angle between the vapour-liquid interface and the glass plates is small. A detailed analysis (not reproduced here) gave us $15 \pm 5^{\circ}$ for the value of this angle. At present, we have no reliable information about the contact angle between the liquid-solid interface and the glass plates.

3.2. Origin of the Convection Flows Surrounding the Bubbles. - It was previously mentioned by Jamgotchian et al. [9] that convection vortices exist in the liquid in the vicinity of gas bubbles. What is the origm of these flows? We investigated this question with the help of micron-size dust particles dispersed in the liquid. When there is no bubble along the growth front, and when the sample is at rest, these particles remain perfectly immobile. When the sample is pulled at a finite velocity $V$, still in the absence of bubble, the dust particles do not exactly follow the translation motion of the sample. They advance towards the growth front at a velocity slightly larger than $V$. This is probably due to the advection flow of the liquid towards the solid, driven by the slight density difference between the two phases. In any case, we confirm the known fact [18] that, in the absence of bubble, no convection flow exist in samples of thickness equal to, or less than, $50 \mu \mathrm{m}$.

In contrast, in the vicnity of a gas bubble, whether at zero or at finite pulling velocity, dust particles are subject to rapid motions revealing the presence of strong convection flows in the 


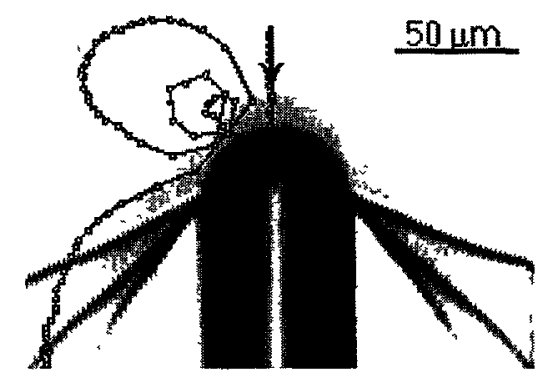

Fig. 4. - Trajectory of a dust particle floating in the liquid near the tip of a tubular bubble in the laboratory reference frame (undoped $\mathrm{CBr}_{4} ; V=32 \mu \mathrm{m} \mathrm{s}^{-1}$ ).

liquid. An example of such a motion in a sample at rest is analysed in Figure 3 over a time period short with respect to the dissolution time of the bubble. This motion is a quasi-periodic rotation, revealing the presence of a vortex whose flow lines are tangent to the bubble interface. Similar vortices exist near the tip of tubular bubbles in samples pulled at constant velocity (Fig. 4). The flow velocity presents a strong maximum near the bubble interface, suggesting that the driving force of the flow is a Marangon effect along this interface. The order of magnitude of the velocity at the interface $\left(>1 \mathrm{~mm} \mathrm{~s}^{-1}\right)$ and the size of the vortex comparable to that of the bubble are compatible with this interpretation.

3.3. Dissolution Kinetics of the Gas Bubbles in the Liquid. - The reason for which the bubbles do not migrate in the liquid while they do so in the solid is not obvious. The origin of the migration of gas bubbles in a condensed phase under the effect of a thermal gradient was explained by Tiller long ago [21]. Basically, it is due to the fact that, since the equilibrium concentrations of the species at an interface depend on the temperature, the concentration inside a bubble placed in a thermal gradient cannot be uniform. Concentration gradients, thus diffusion flows, exist in the bubble, and drag the interface along. The fact that the bubbles do not migrate in the liquid may simply be due to the pinning of the solid-vapour-glass contact lines by the imperfections of the glass plates, but a detailed analysis (not attempted here) would have to take account of other factors, in particular, the attenuation of the thermal and concentration gradient due to the convection flows.

The slow dissolution of the bubbles with time is essentially controlled by the diffusion of the gas in the surrounding unsaturated liquid. For obvious reasons (modification of the diffusion field by the capllary walls and the other bubbles, convection flows), the measured time evolution of the bubble diameters cannot be expected to follow exactly the $(D t)^{-1 / 2}$ law ( $D$ : diffusion coefficient; $t$ : time) characteristic of a diffusion-driven kinetics. A typical example of an initially large bubble is shown in Figure 5. The bubble shrinkage is much slower than that predicted by a $(D t)^{-1 / 2}$ law, except in its terminal stage, when the bubble diameter is smaller than about $30 \mu \mathrm{m}$. In the case of Figure 5 , the bubble even begins by growing because it is absorbing the gas coming from a smaller near-by bubble (Ostwald ripening). So, the annealing time necessary for the bubbles to disappear is very sensitive to their size and their distribution along the capillary. This time is typically 1 minute for most of the bubbles, whose diameters rarely exceed $50 \mu \mathrm{m}$, but can be more than 1 hour for bubbles of diameter comparable to the width of the capllary. We therefore generally discarded the samples containing such large bubbles (an exception to this rule is mentioned in Sect. 7.2). 


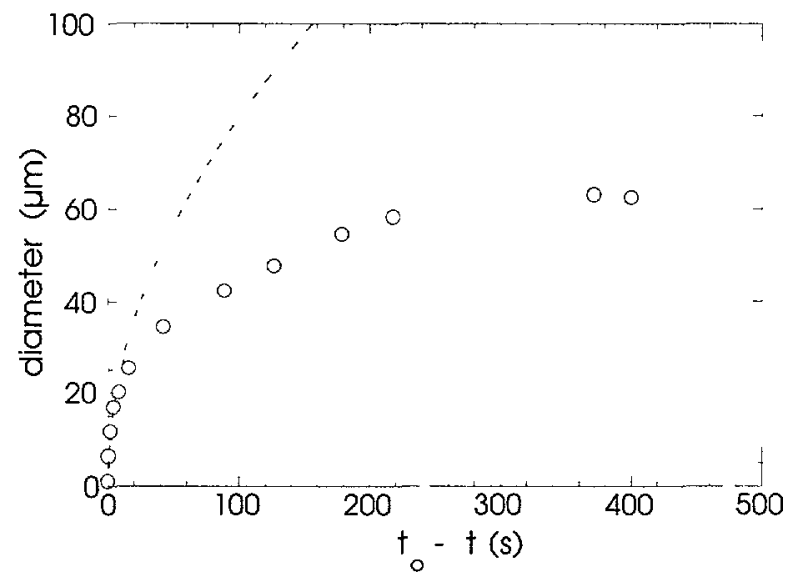

Fig. 5. - Diameter of a bubble in the liquid at rest as a function of $t_{0}-t\left(t_{0}\right.$ : time at which the bubble finally disappeared). Dotted line: $\left[D\left(t_{0}-t\right)\right]^{-1 / 2}$ with $D=10^{-10} \mathrm{~cm}^{2} \mathrm{~s}^{-1}$

\section{Effects of Dissolved Residual Impurities}

4.1. REsidual-IMPURITY CONTENT OF UNDOPED $\mathrm{CBr}_{4}$. - A well-known in-situ method of determining some of the physical properties of alloys intervening in their solidification (partition coefficient $K$, liquidus slope $m$, diffusion coefficient in the liquid $D$ ) is to measure both the cellular-threshold velocity $V_{\mathrm{c}}$ and the initial-recoll curve of the alloy at velocities lower than $V_{c}$. We previously applied this method to the determination of the physical properties of the binary system $\mathrm{CBr}_{4}{ }^{-} \mathrm{C}_{2} \mathrm{Cl}_{6}$ [10]. To do this, we had to correct the measurements for residual-impurity effects in a way discussed later on in this Section. We found $K^{\mathrm{H}} \approx 0.75$, $m^{\mathrm{H}} \approx 70 \mathrm{~K}$ and $D^{\mathrm{H}} \approx 5 \times 10^{-10} \mathrm{~m}^{2} \mathrm{~s}^{-1}$, where $\mathrm{H}$ stands for hexachloroethane. Here, we report the results for the same type of measurements carried out on samples of undoped $\mathrm{CBr}_{4}$. Such measurements were possible because bubble nucleation did not occur during the initial transient of the samples. This absence of "spontaneous' bubble nucleation is an important feature of our material, to which we shall return in Section 5 .

For clarity, let us recall some well-known formulae. Consider first the case of the binary system $\mathrm{CBr}_{4}-\mathrm{X}$. According to the constitutional-supercooling (CS) approxımation (certainly sufficiently accurate for our purpose), the cellular-threshold velocity $V_{\mathrm{c}}^{\mathrm{X}}$ reads:

$$
V_{\mathrm{c}}^{\mathrm{x}} \approx V_{\mathrm{CS}}^{\mathrm{X}}=\frac{G D^{\mathrm{X}}}{\Delta T_{0}^{\mathrm{X}}}
$$

where $\Delta T_{0}^{\mathrm{X}}$ is the thermal gap of the alloy, given by

$$
\Delta T_{0}^{\mathrm{X}}=m^{\mathrm{x}}\left(K^{\mathrm{x}^{-1}}-1\right) C_{\infty}^{\mathrm{X}}
$$

For a ternary system $\mathrm{CBr}_{4}-\mathrm{A}-\mathrm{X}$, where $\mathrm{A}$ stands elther for the addition element $\mathrm{H}$ or a second impurity (contaminant) $\mathrm{X}^{\prime}$, it reads

$$
V_{\mathrm{c}}^{\mathrm{X}} \approx \frac{G}{\Delta T_{0}^{\mathrm{X}} / D^{\mathrm{X}}+\Delta T_{0}^{\mathrm{A}} / D^{\mathrm{A}}}
$$




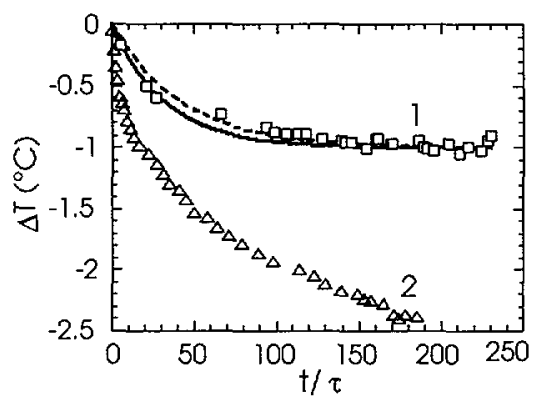

Fig. 6. - Initial recoil of the front recorded in two different samples of undoped $\mathrm{CBr}_{4}$ at $V=$ $8.2 \mu \mathrm{m} \mathrm{s}^{-1}$ as a function of the reduced time $t / \tau$ (see text). " 1 ": non-contaminated sample. "2": strongly contaminated sample. Continuous lıne: WL approximation with $K^{\mathrm{X}}=0.022$. Broken line: STR approximation with $K^{\mathrm{X}}=0.024$.

The recoil of the front is defined as $\Delta T(t)=T-T_{0}$, where $T$ is the temperature of the front at time $t$ and $T_{0}$ the temperature of the front at rest $(\Delta T(t)$ is therefore negative). When $t \rightarrow \infty,|\Delta T(t)|$ tends to a constant value $\Delta T_{0}$ (the amplitude of the recoil), which is equal to $\Delta T_{0}^{\mathrm{X}}$ for a binary alloy, and to

$$
\Delta T_{0}=\Delta T_{0}^{\mathrm{X}}+\Delta T_{0}^{\mathrm{A}}
$$

for a multi-component dilute alloy. Except for the asymptotic value $\Delta T_{0}$, there exists no explicit formula for $\Delta T(t)$. It is common practice to assume that $\Delta T(t)$ is approximately an exponential, and to assimilate the characteristic time of the exponential to the approximate value of the terminal time of the recoil given by Smith, Tiller and Rutter (STR) [15]:

$$
\tau_{\mathrm{r}}^{\mathrm{X}} \approx \tau_{\mathrm{STR}}^{\mathrm{X}}=\frac{\tau^{\mathrm{X}}}{K^{\mathrm{X}}}
$$

where $\tau^{\mathrm{X}}=D^{\mathrm{X}} / V^{2}$ is the diffusion time for $\mathrm{X}$. The validity of this formula has been questioned recently. Using a different approximation, Warren and Langer (WL) found that equation (5) is only valid in the limit $K^{\mathrm{X}} \rightarrow 0$ [22]. Performing the numerical integration of the equations of the mitial transient, Caroli et al. obtained the exact values of $\Delta T(t)$ in a series of cases, and compared them with those obtained with the help of the STR and WL approximations [23]. They found that the WL approximation is generally excellent, while the STR approximation Is seriously questionable, especially as concerns the behaviour of $\Delta T(t)$ at $t \rightarrow 0$ and $t \rightarrow \infty$. The measurements presented here are quite inaccurate precisely in these two ranges. So, in our case, the STR procedure gives practically the same results as the numerical fit of the exact or the WL curves onto the measured curve (this is shown in Fig. 6). We therefore generally analysed the initial-recoil curves according to the STR formula. Similarly, in the case of our multi-component samples, the approximation $\Delta T(t) \approx \Delta T^{\mathrm{X}}(t)+\Delta T^{\mathrm{A}}(t)$ (which is exact in the limit $t \rightarrow \infty)$ is acceptable. We checked this numerically with the help of a WL-type approximation extended to multi-component dilute alloys (unpublished results).

Figure 6 shows two typical recoil curves measured at the same pulling velocity in two different samples of undoped $\mathrm{CBr}_{4}$. These curves have been corrected for the instrumental transient as explained in Section 2. The reduced time appearng in abscissa is the actual time divided by a conventional diffusion time, which we took equal to $\tau^{\mathrm{H}}=D^{\mathrm{H}} / V^{2}$. Curve 1 corresponds to a so-called non-contaminated sample, and Curve 2 to a strongly contaminated sample. In the non-contaminated sample, the initial recoil is well completed before the end of the experimental run, while, in the contaminated one, it continues until the end of the run. Most of our samples 
were slightly contaminated, and presented initial-recoil curves intermediate between the two extreme types shown in Figure 6.

Let us now turn to the quantitative discussion of these results.

4.1.1. Non-Contaminated Undoped Samples. - In Figure 6, the measured initial-recoil curve of the non-contaminated sample is compared to the initial-recoil curve of a binary alloy calculated following the WL approximation. The two curves are reasonably similar, justifying the assimilation of undoped $\mathrm{CBr}_{4}$ to a binary alloy. The values introduced in the calculation are $\Delta T_{0}^{\mathrm{X}} \approx 1 \mathrm{~K}$ and $K^{\mathrm{X}} \approx 0.022$ (the value of $K^{\mathrm{X}}$ found with the help of the STR approximation is 0.024). The other non-contaminated samples gave the same values to within $20 \%$. On the other hand, we found $V_{\mathrm{c}}^{\mathrm{X}}=6 \pm 2 \mu \mathrm{m} \mathrm{s}^{-1}$ [24]. Substituting these values into the above equations, we obtain $D^{\mathrm{X}} \approx 7.5 \times 10^{-10} \mathrm{~m}^{2} \mathrm{~s}^{-1}$ and $m^{\mathrm{X}} C_{\infty}^{\mathrm{X}} \approx 0.02 \mathrm{~K}$ (note that the displacement of the front at rest due to $\mathrm{X}$, given by $m^{\mathrm{X}} C_{\infty}^{\mathrm{X}} / G$, is about $2 \mu \mathrm{m}$, and is therefore hardly detectable with the microscope). It will be seen in Section 6 that an independent method of measurement leads to $C_{\infty}^{\mathrm{X}} \approx 5 \times 10^{-4}$ and thus to $m^{\mathrm{X}} \approx 50 \mathrm{~K}$.

4.1.2. Contaminated Undoped Samples. - The origin of the contamination is not known with certainty. It does not seem to originate from the zone-melting process (the two samples in Fig. 6 were filled with $\mathrm{CBr}_{4}$ from the same zone-refined rod). It is probable that most of the contamination stems from an imperfect cleanness of the container walls. The chemical nature of the contaminants is also uncertain. The very long duration of the initial recoil (also observed on the solid-vapour triple points in contaminated undoped samples; see Sect. 4.2.4) indicates that one of the contaminant species (noted $X^{\prime}$ thereafter) has a very small partition coefficient $\left(K^{\mathrm{X}^{\prime}} \ll K^{\mathrm{X}}\right)$. It must however be noted that the initial recoil does not only last longer in the contaminated sample than in the non-contaminated ones, it also starts more abruptly. This indicates that there is also a contaminant species having a partition coefficient of the same order of magnitude as $K^{\mathrm{X}}$

4.2. Residual Impurities in Doped Samples. - The addition of about $3 \mathrm{~mol} \% \mathrm{C}_{2} \mathrm{Cl}_{6}$ does not significantly change the residual-impurity content of the samples. Like the undoped ones, all the doped samples contain a relatively invariable amount of the residual gas $\mathrm{X}$, and most of them additionally contain a certain, unpredictable amount of contaminant. On the other hand, in the doped samples, residual-impurity effects occur in combination with the effects due to the main solute, and the question is to separate these two types of effects. Four different issues must be considered in turn.

4.2.1. Initial Recoil $\left(V<V_{c}\right)$. - In reference [10], the initial-recoil curves of samples with various $\mathrm{C}_{2} \mathrm{Cl}_{6}$ concentrations were measured in order to gain information about the phase diagram of the binary system $\mathrm{CBr}_{4}-\mathrm{C}_{2} \mathrm{Cl}_{6}$. Two curves were measured simultaneously in a multi-capillary sample, one corresponding to a doped alloy and the other to an undoped one (an example is given Fig. 7). We subtracted the latter from the former and considered the resulting "corrected" curve as a good approximation of what would have been the initial-recoil curve of the binary $\mathrm{CBr}_{4}-\mathrm{C}_{2} \mathrm{Cl}_{6}$ alloy in the absence of impurity. The validity of this procedure clearly follows from the above conclusions. The fact that, in Figure 7, the corrected curve does not saturate at times much larger than $\tau^{\mathrm{H}}$ is certainly due to the contamination. The small amplitude of this residual long-lasting recoil indicates that the contamination level was low in both capillaries.

4.2.2. Shift of the Cellular-Threshold Velocity. - In reference [10], in order to determine the diffusion coefficient of $\mathrm{C}_{2} \mathrm{Cl}_{6}$ in the liquid, we plotted $1 / V_{\mathrm{c}}$ as a function of $\Delta T_{0}$, fitted a straight line onto the experimental points, and assumed that the reciprocal of the slope of this line was 


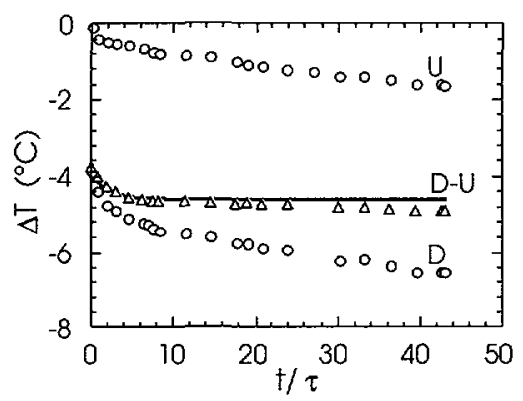

Fig. 7. - Initial-recoil curve of an undoped $(U)$ and a doped $(D)$ sample measured simultaneously in a multi-capillary sample at $V=5.1 \mu \mathrm{m} \mathrm{s}^{-1}$. The temperature corresponding to $\Delta T=0$ is that of the front at rest in the undoped sample. $D-U$ : difference between data $D$ and data $U$. Continuous line: WL approximation with $K^{\mathrm{H}}=0.75$.

equal to $D^{\mathrm{H}} / G$. In doing so, we were presuming that $D^{\mathrm{X}} \approx D^{\mathrm{H}}$, and, much more seriously, we were neglecting the shift of the observed value of $V_{c}$ with time due to the slow, progressive accumulation of $\mathrm{X}$ at the interface. This point is worth considering in detail. In any experiment devoted to the determination of $V_{c}$, it is necessary to perform velocity jumps from below to above $V_{\mathrm{c}}$ and vice versa. The characterıstic time for the formation/disappearance of the cells after the jump is, typically, $5 \tau^{\mathrm{H}}$ (see the next item). Since the time-scale $\tau^{\mathrm{X}}$ of the accumulation of $\mathrm{X}$ is much larger than $\tau^{\mathrm{H}}$, the variation of $C^{\mathrm{X}}$ taking place during the formation of the cells can be neglected, in a first approach. The velocity at which the transformation is actually observed is thus approximately given by a quasi-stationary version of equation (3), in which the terminal value $\Delta T_{0}^{\mathrm{X}}$ of the recoil due to $\mathrm{X}$ is replaced by its instantaneous value $\Delta T^{\mathrm{X}}(t)$. It is then evident that the measured value $V_{c}$ depends on the previous history of the sample. This is probably the main cause of the relatively large scatter of the experimental points in the $V_{\mathrm{c}}^{-1}-\Delta T_{0}$ diagram in reference [10]. Incidentally, it also follows from this analysis that the existing reports on the direct or indirect nature of the cellular bifurcation should perhaps be reconsidered. Suppose, for instance, that the experımental procedure applied to determine the nature of the bifurcation consists of first increasing, and then decreasing $V$ step by step, and to compare the "upward" threshold velocity $V_{+}$to the "downward" one $V_{-}$. It is obvious that the presence of a small- $K$ residual impurity could suffice to make the last-measured value of $V_{\mathrm{c}}$, namely, $V_{-}$, appear noticeably smaller than the first-measured one, $V_{+}$, irrespective of the nature of the bifurcation.

4.2.3. Time Evolution of the Cell Tips and Groove Bottoms $\left(V>V_{\mathrm{c}}\right)$. - The quantitative descruption of the effects of residual impurities on the morphology of the cellular/dendritic fronts in doped samples at $V>V_{\mathrm{c}}$ cannot be undertaken in the frame of this article. However, the general trends are simple, and can be indicated here. Consider, for instance, the initial transient of a doped sample at a velocity noticeably above the cellular threshold $\left(V>2 V_{\mathrm{c}}\right)$. This transient is divided in a first stage lasting a few $\tau^{\mathrm{H}}$, during which the cells (or dendrites) are formed, and a second stage of duration time of the order of $\tau^{\mathrm{X}}$, corresponding to the accumulation of $\mathrm{X}$ at the interface. This accumulation provokes a long-lasting recoil of the front. The interesting point is that this recoil is not the same near the cell tips and in the intercellular grooves. Roughly speaking, the long-lasting recoil of the cell tips is negligible, while the depth of the intercellular grooves increases considerably with time, indicating that most of the impurities accumulate in the previously-formed grooves. Simultaneously, the shape of the front in the region of the groove bottom changes considerably. 


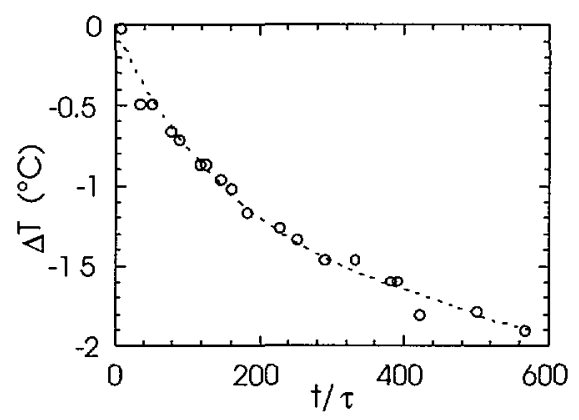

Fig. 8. - Recoil curve of the solld-liquid-vapour triple point in an undoped, highly contaminated sample at $V=34.5 \mu \mathrm{m} \mathrm{s}^{-1}$ (the broken line is a guide for the eyes).

4.2.4. Recoil of the Triple Points in Contaminated Samples. - A method to test the quality (i.e., the level of contamination) of the samples complementary to measure the initial-recoil curve is to follow up the temperature of some particular point of the front during the course of the solidification run. This method is especially useful when triple points are present along the growth front, as in eutectics or in the tubular-bubble regime. In the case of tubular bubbles in uncontaminated undoped samples, the temperature $T_{\theta}$ of the solid-liquid-vapour triple points is given by $T_{\theta}=T_{\mathrm{B}}+\Delta T_{\mathrm{GT}}$, where $\Delta T_{\mathrm{GT}}=a_{0} \kappa$ is the Gibbs-Thomson capillary term, $a_{0}$ is the capillary constant $(\approx 0.09 \mathrm{~K} \mu \mathrm{m}$ for the solid), $\kappa$ is the curvature of the interface. In general, $\kappa$ is small enough for $\Delta T_{\mathrm{GT}}$ to be negligible, and $T_{\theta}$ should thus seem invariable. In fact, during long solidification runs at constant $V, T_{\theta}$ is observed to recoil with time as shown in Figure 8. This long-lasting "triple-point recoil" reveals the presence of a contaminant. In doped samples, the presence of an additional component in principle relaxes the condition $T_{\theta}=T_{\mathrm{B}}+\Delta T_{\mathrm{GT}}$ even in the absence of contamination, but triple-point recoils of similar amplitudes and duration times as in undoped samples are nevertheless observed.

\section{Nucleation of the Gas Bubbles}

The undercooling of the liquid and the nature of the foreign particles (either floating in the liquid or attached to the walls of the container) serving as nucleation substrate are the two main factors controlling (heterogeneous) nucleation. To each nucleation substrate is attached a critical value $\Delta T_{\text {nucl }}$ of the undercooling at which nucleation takes place (almost) immediately. In the case of gas bubbles, the high compressibility of the vapour entails that the spatial variations of the pressure connected with the advection-convection flows can also play a role. We observed a number of nucleation events in doped and in undoped samples of various contamination level, and found that they most generally obeyed the following rules:

- bubble nucleation takes place in the intercellular grooves, at an undercooling of the liquid at least equal to $4^{\circ} \mathrm{C}$;

- bubble nucleation never occurs during an mitial transient, nor in a steady state at constant $V$. It only occurs during the transients following upward velocity jumps (an exception is mentioned in footnote 1 ).

The interpretation of these observations would be straightforward if it was moreover observed that nucleation took place at the point of maximum undercooling of the liquid, 1.e., at the bottom of the intercellular grooves. If such were the case, we could conclude that the first active nucleation substrates have a value of $\Delta T_{\text {nucl }}$ of about $4^{\circ} \mathrm{C}$, and that such substrates are 


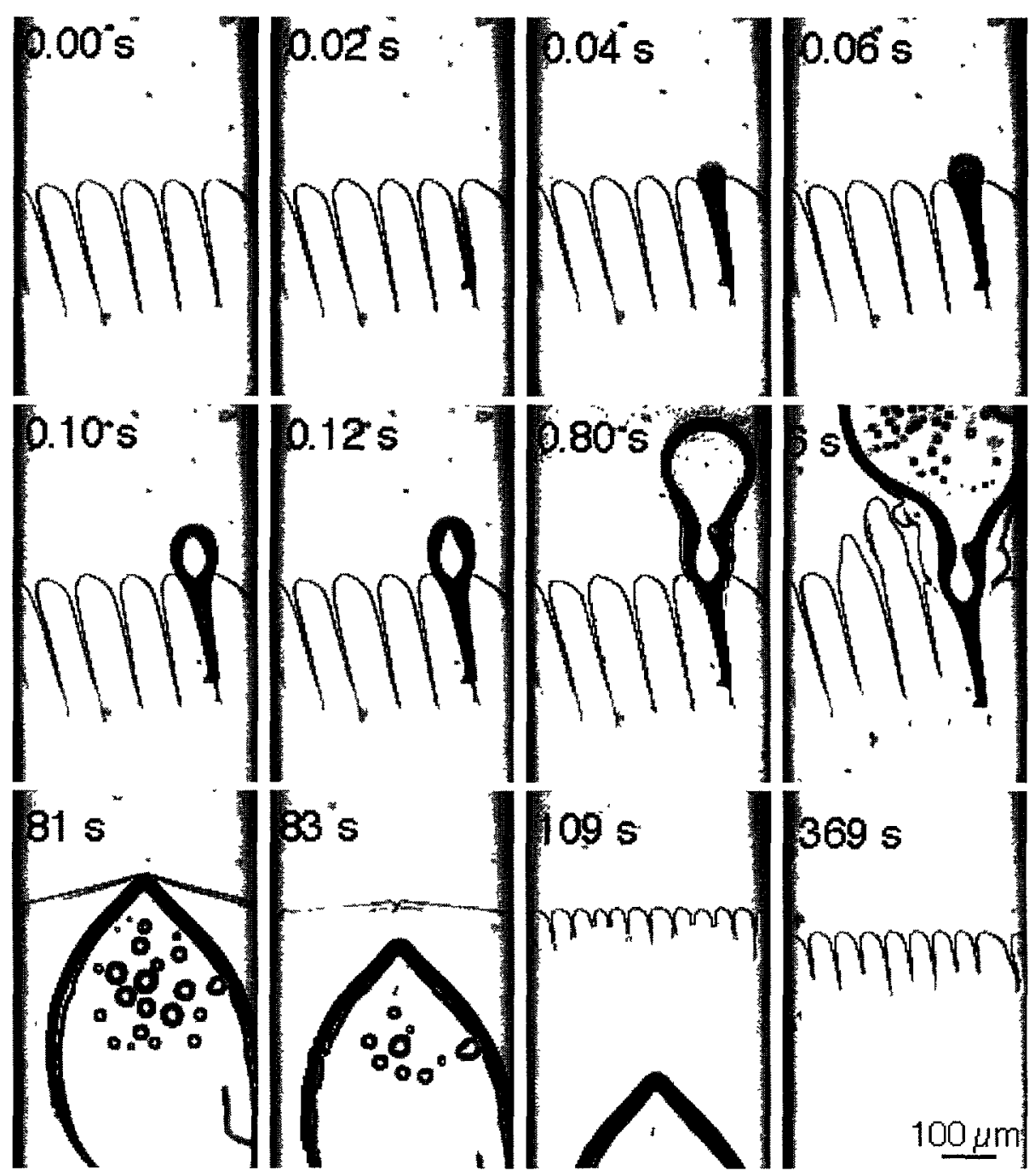

Fig. 9. - Doped sample. Snapshots of a bubble at various stages of the nucleation-growth process. The times indicated in the upper left corners of the photographs are counted from the moment of the nucleation. A velocity jump from 5.6 to $10.7 \mu \mathrm{m} \mathrm{s}^{-1}$ was appled at $-53 \mathrm{~s}$ (see Fig. 10).

In sufficient number for nucleation to occur as soon as the undercooling at some point of the front reaches $4{ }^{\circ} \mathrm{C}$. In reality, things are somewhat more complicated.

Figure 9 shows an experıment performed in a doped sample, in which a bubble-nucleation event was triggered by an upward velocity jump. Figure 10 shows the time evolution of the temperature of some remarkable points of the front (cell tips, groove bottoms, bubble tip and triple points) during this experiment run. The sample was first pulled a long time at $5.6 \mu \mathrm{m} \mathrm{s}^{-1}$, and had reached the steady state at this velocity when $V$ was suddenly switched to $10.7 \mu \mathrm{m} \mathrm{s}^{-1}$. The nucleation of a bubble (taken as origin of time in Figs. 9 and 10) occurred some $50 \mathrm{~s}$ after the jump. In this experıment, the bubble had a finite lifetime. It disappeared about $80 \mathrm{~s}$ after its nucleation, "pinched-off" by the growng solid. The growth 


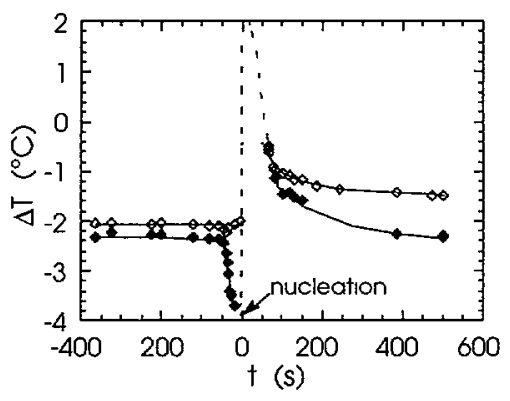

Fig. 10. - Time evolution of the temperature of some particular points of the growth front during the same experimental run as in Figure 9. Open diamonds: cell tips. Closed diamonds: groove bottoms. Dash-dot line: bubble tip. Broken line: triple point.

front then relaxed towards the steady state without bubble at $10.7 \mu \mathrm{m} \mathrm{s}^{-1}$. In agreement with the remarks made in Section 4.2 .3 , the cells are re-formed after a time of order $\tau^{\mathrm{H}}(\approx 5 \mathrm{~s})$ after the pinching-off, and rapidly practically reach their stationary position while the groove depth continues to increase at a much slower rate. A simular experiment performed in an undoped sample is shown in Figure 11. Contrary to the preceding one, it does not lead to the elimination of the bubble but to its stabilisation in the form of a stationary tubular bubble.

The bubble-growth process subsequent to the nucleation and the reason of the difference between the final fates of the bubble in these two experiments are discussed in the next Section. For now it should simply be noted that the nucleation of the bubble takes place during (and interrupts) the transitory rise of the groove depth subsequent to the velocity jump (Fig. 10). The occurrence of such a transient is not a surprise. A long time ago, Smith et al. showed theoretically that a sudden velocity jump applied to a sample which has previously reached its steady state at $V_{1}$, is followed by a transitory rise of the solute concentration - thus of the recoll of the front - above the steady-state value [15]. The characteristic duration time of the transient is $D^{\mathrm{X}} /\left(K^{\mathrm{X}} V_{1} V_{2}\right)(\approx 500 \mathrm{~s}$ in our case). For a given amplitude of the velocity jump, the maximum value reached by $C^{\mathrm{X}}$ increases as $K^{\mathrm{X}}$ decreases. With $K^{\mathrm{X}}$ of the order of $10^{-2}$, a jump of relatively modest amplitude suffices to provoke a considerable transitory extra-recoil. This is in agreement with our observations, which moreover show that, when the front is cellular, the transitory extra-recoll is mostly concentrated in the grooves.

The shape of the incipient bubbles in Figures 9 and 11 are similar. They clearly bring to light the 3D nature of the cellular pattern. In particular, they present a small lateral excrescence partly overlapping one of the neighbouring cells. This excrescence is close to their coldest point, which, in both experiments, is at an undercooling of about $4{ }^{\circ} \mathrm{C}$. The grooves however extent down to larger undercoolings (this does not appear clearly in Fig. 11 because of the weak contrast of the $3 \mathrm{D}$ grooves, but was evident under the microscope). There are two possible explanations of this fact.

The critical nucleus itself is of course invisible with the microscope but we can deduce from the order of magnitude of the initial growth rate of the bubble $\left(10^{4} \mu \mathrm{m} \mathrm{s}^{-1}\right)$ that nucleation occurred about $0.01 \mathrm{~s}$ before we see $1 \mathrm{t}$. The first possibility is that the bubbles have in fact nucleated at the bottom of the grooves but have jumped forward under the effect of the capillary forces (see Fig. 1 in Ref. [9]) during this $0.01 \mathrm{~s}$. The above-mentioned lateral excrescence would then be the site, not of nucleation, but of attachment of the bubble onto the solid. The other possibility is of course that the lateral excrescence is the nucleation site itself, the 

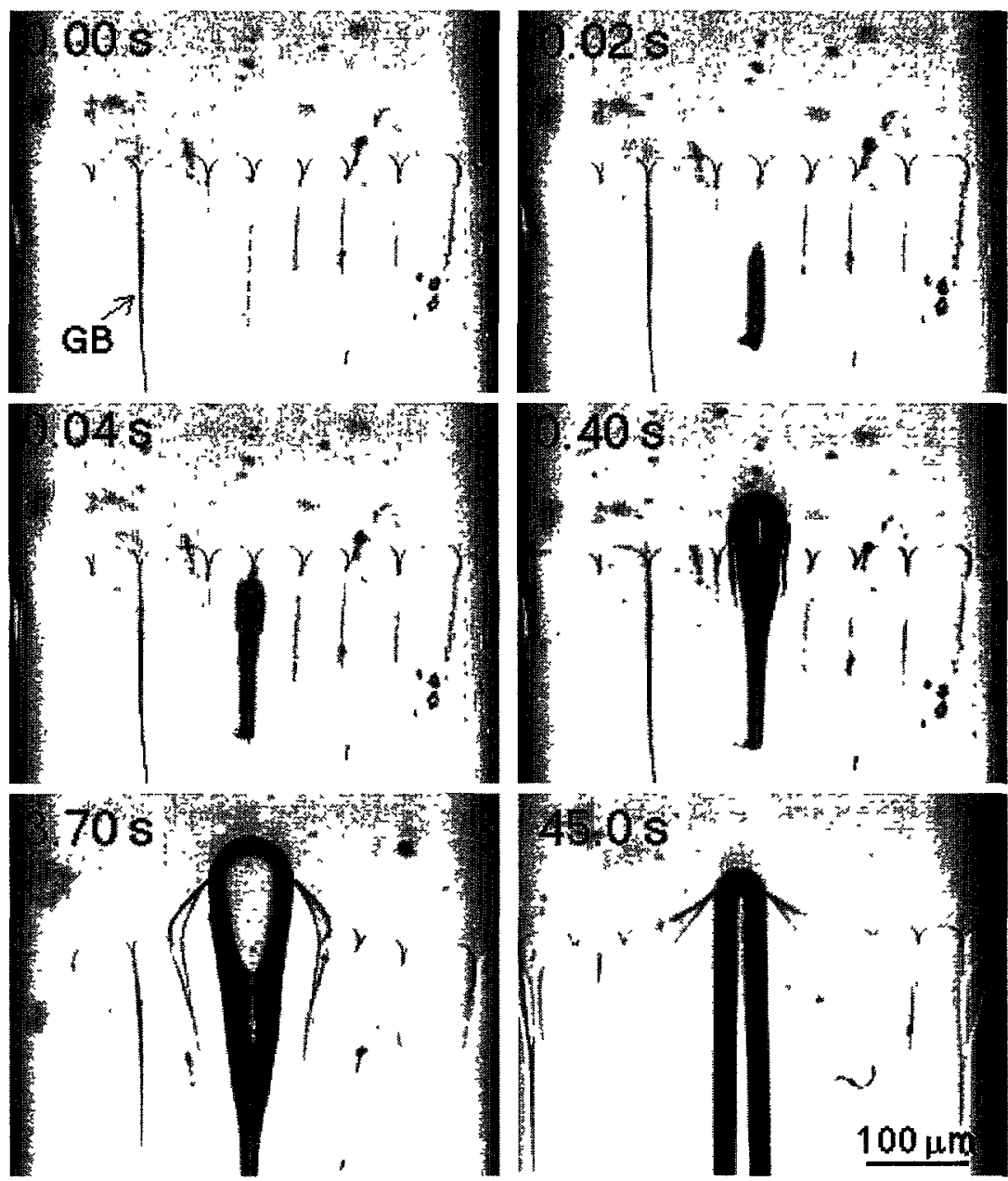

Fig. 11. - Undoped sample. Snapshots of a bubble at varıous stages of the nucleation-growth process. Time: as in Figure 9. A velocity jump from 8.2 to $32 \mu \mathrm{m} \mathrm{s}^{-1}$ was applied at $-29 \mathrm{~s}$. GB: grain boundary.

existence of liquid pockets (between the nucleation site and the groove bottoms) in which no nucleation occurs although the temperature is lower than $T_{\text {nucl }}$ being explained by a very low total foreign-particle density, and (or) by the fact that most foreign-particles are entrapped by the solid before reaching the groove bottoms. The reproducible appearance of this particular nucleation site could then be connected with the shape of the flow lines in this region of the liquid.

\section{Solid-Vapour Coupled Gowth}

6.1. Initial Stages. - We pointed out above that the experimental run of Figure 9 leads to the elimmation of the bubble, while that of Figure 11 ends with a stationary tubular bubble. What are the reasons of these different outcomes of the two experıments? We shall show in the next Section that a stationary tubular bubble can exist at the same velocity $\left(10.7 \mu \mathrm{m} \mathrm{s}^{-1}\right)$ and 


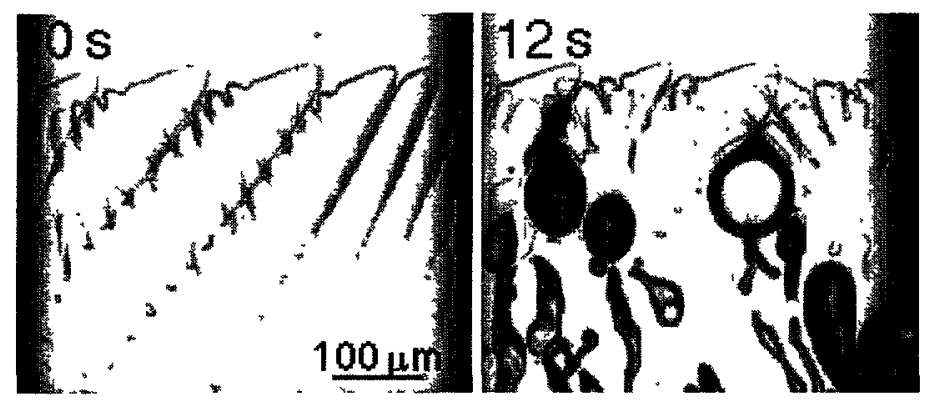

Fig. 12. - Strongly contaminated doped sample at $V=32 \mu \mathrm{m} \mathrm{s}^{-1}$ Onset of the "boiling front" regime.

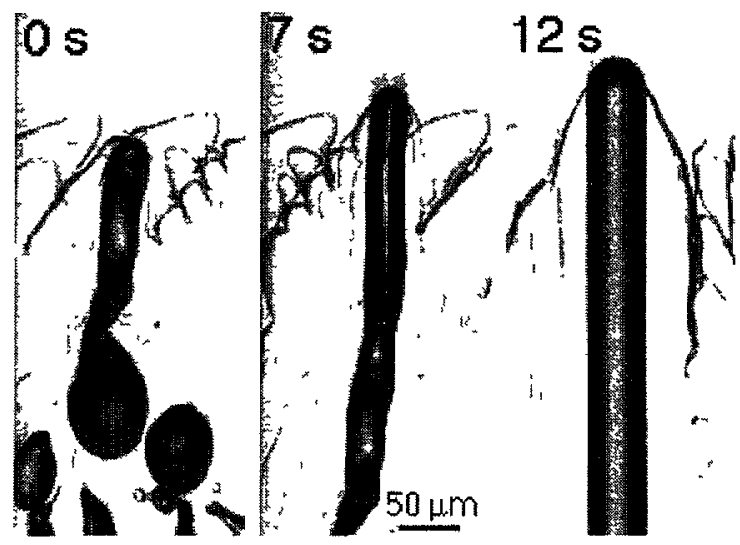

Fig. 13. - Same run as in Figure 12, at a later stage of the process.

composition (doped sample) as those at which a pinching-off is observed in Figure 9 (the lateral width of such a bubble would be about $50 \mu \mathrm{m}$ ). Thus the ultimate fate of the bubble is not uniquely determined by the composition of the alloy and the pulling velocity. It also (in fact, mostly) depends on the particular environment in which nucleation takes place. A detailed examination of the intial stages of the growth of the bubbles will allow us to substantiate this view.

The growth process of a bubble subsequent to its nucleation obviously comprises a first stage during which the bubble grows confined in an intercellular groove, and a second one during which the bubble tip evolves in the liquid far ahead of the remainder growth front. Durng the first stage, the advancing bubble tip creates a depletion of solute(s) in the surrounding liquid, which in turn causes the solid to grow in the direction of the bubble.

The bubble and the surrounding solid are thus in a tight competition, the outcome of which may be quite different in different runs at the same alloy composition and pulling velocity. In Figures 9 and 11, the bubble tip has a large growth velocity $\left(>100 \mu \mathrm{m} \mathrm{s}^{-1}\right)$ at the moment where it reaches the level of the cell tips. This is not always the case, as shown in Figures 12 and 13. In Figure 12, the bubbles are entrapped by the solid before reaching the cell-tip level. In Figure 13, the bubble reaches the cell-tip level with a velocity hardly larger than $V$, and immediately takes on 1 ts stationary tubular form. Obviously, these striking differences of 

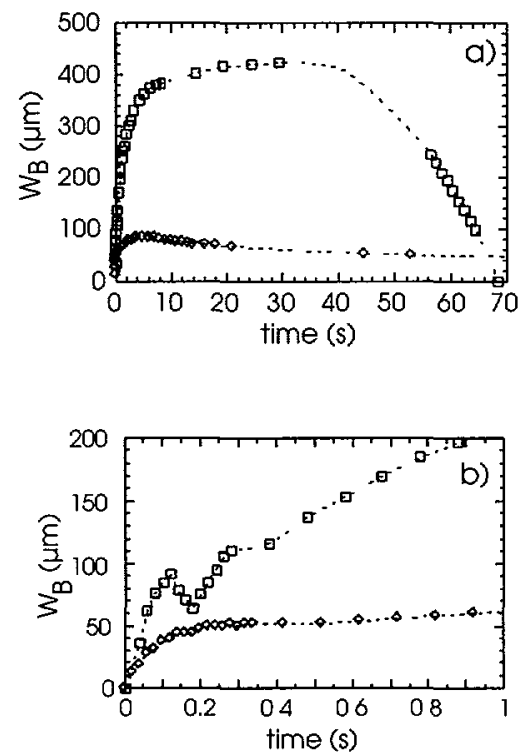

Fig. 14. - a) Time evolution of the lateral width of the bubble. b) Enlarged view of the initial stage of (a). Open squares: experimental run of Figure 9. Open diamonds: experimental run of Figure 11.

behaviour are connected with differences in the geometry of the solid in the region of the groove bottom, i.e., in the so-called mushy zone. This geometry in turn depends on the orientation of the crystal with respect to the solidification setup (defined by three independent variables [14]) and the sample thickness. Roughly speaking, an open structure of the mushy zone (as in Figs. 9 and 11) allows the bubble to jump rapidly into the liquid, while a closed structure leads to the early entrapping of the bubble (as in Figs. 12 and 13, in which the particular orientation of the $\langle 100\rangle$ dendritic axes results in the overlap of the grooves by the dendritic sidebranches).

At the onset of the second stage of the growth (if there is one), a local structure has formed, which we shall henceforth call solid-vapour (SV) finger. The tip of this structure consists of a cap of liquid-vapour interface limited by a solid-liquid-vapour contact line. We have reproduced in Figure 14 the time evolution of the lateral width $W_{\mathrm{B}}$ of the bubble (most of the time equal to the distance between the two triple points) durıng the two experiments described in Figures 9 and 11 . This evolution clearly has an oscillatory nature - also deducible from the pear-like shape of the bubbles - but there is a large difference in the amplitude of the oscillations between the Figures 9 and 11. This difference can be understood by reference to previous observations made in other types of directionally-solidified systems.

In dendritic fronts, it has recently been noticed that, when a new dendrite is created as the result of the so-called tail instability, this dendrite first osc1llates in the same way as the SV finger observed in Figure 11 [14]. In general terms, this oscillation is explained by the fact that the position and the velocity of a part of the growth front are independent variables (they depend on the concentration and the concentration gradient in the liquid at the front, respectively). The growth velocity of the new structure being larger than $V$ when it first reaches the steady-state position, it is bound to grow past this point, until it is slowed down by the increasing temperature of its tip. Most probably, the oscillations of the SV fingers that we observe here have essentially the same origin. 
In two-dimensional lamellar eutectic growth fronts, there exists a type of oscillations, called "giant oscillations" [25], with which the large-amplitude oscillations observed in Figure 9 clearly present a profound analogy. It can be seen in Figure 14 that, during most of the giant-oscillation process, the distance between the two triple points is so large that they do not "feel" each other. In other words, each triple point essentially acts as a quas1-stationary local structure travelling with a forward velocity practically equal to $V\left(=10.7 \mu \mathrm{m} \mathrm{s}^{-1}\right)$ and a lateral velocity $v_{\mathrm{B}}$ of the order of $50 \mu \mathrm{m} \mathrm{s}^{-1}$ On arriving at a distance from an obstacle of the order of the lateral diffusion length $D / v_{\mathrm{B}}(\approx 10 \mu \mathrm{m})$, these travelling triple points slow down, stop, and then generally start again in the opposite direction. In the present case, a first "rebound" of the triple points is observed at $t \approx 0.2 \mathrm{~s}$, when the two triple points are running towards each other, and a second one at $t \approx 30 \mathrm{~s}$, when they approach the walls of the capillary. The third rebound, which one expects to occur at $t \approx 70 \mathrm{~s}$, is replaced by the merging of the triple points and the disappearance of the bubble. The occurrence of this pinching-off event is not altogether surprising, although we do not understand it in detail, since we know, from the study of the dynamics of lamellar eutectics that this is one of the generic possibilities of the system.

In summary, we have identified three possible fates for a bubble nucleating at a solidification front: a giant oscillation resulting in a pinching-off, a damped oscillation leading to a stationary tubular state, and an immediate entrapment inside the mushy zone. The selection of one of these modes of growth $\left(^{1}\right)$ by the system essentially depends on the velocity of the bubble tip at the end of the first stage of growth, itself determined by the structure of the solid in the mushy zone. We naturally do not claim this list to be exhaustive. In particular, it does not include the case - common, for instance, in metallurgy [2] — of large solidification velocities, in which the temperature of the dendritic tips is larger than that of the solid-liquid-vapour triple points.

6.2. The Steady Tubular Pattern. - In this Section, we present a brief study of the evolution of the morphology of the front in the stationary tubular regime as a function of the growth velocity. The questions at stake are the validity, and the limits, of the analogy between this growth pattern and lamellar eutectic patterns, and, from a more practical standpoint, the importance of the perturbation caused by a tubular bubble on the remainder of the front. For the latter purpose, this part of the study has been carried out mostly with doped $\mathrm{CBr}_{4}$. We utilised two different experimental procedures. In one of them, we trigger a bubble nucleation by a jump at some, necessarily large velocity, and then decreased the velocity step by step, waiting at each velocity for the bubble to reach its stable configuration. In the second method, we utilised samples intially containing large gas bubbles which did not dissolve during the prelimmary annealing of the sample. In this case, the passage of the growth front over the bubbles gives rise to a tubular bubble even at low velocity. We could therefore start at a low initial velocity, and then increase the velocity step by step. Both methods gave the same results, as will be seen presently.

Figure 15 shows images of a tubular bubble obtained by the second method, at several different values of $V$. The stationary nature of the pattern is made obvious by the regularity of the diameter of the vapour tubes left behind in the solid. Contrary to Jamgotchian et al. [9], we observe no detectable oscillations of the bubble diameter. Indeed, oscllations are

$\left({ }^{1}\right)$ The first and the third scenarios obviously give rise to repeated nucleations, i.e., to more or less periodic regimes. In the case of Figure 18 , the sample was so strongly contaminated that bubble nucleation took place in the steady-state at the velocity considered $\left(32 \mu \mathrm{m} \mathrm{s}^{-1}\right)$ A peculiar type of solid-vapour mode of growth ensued, consisting of a rapid, erratic repetition of the nucleation/trapping process within the mushy zone. This is probably the regime previously called "boiling front" by some authors [4]. 

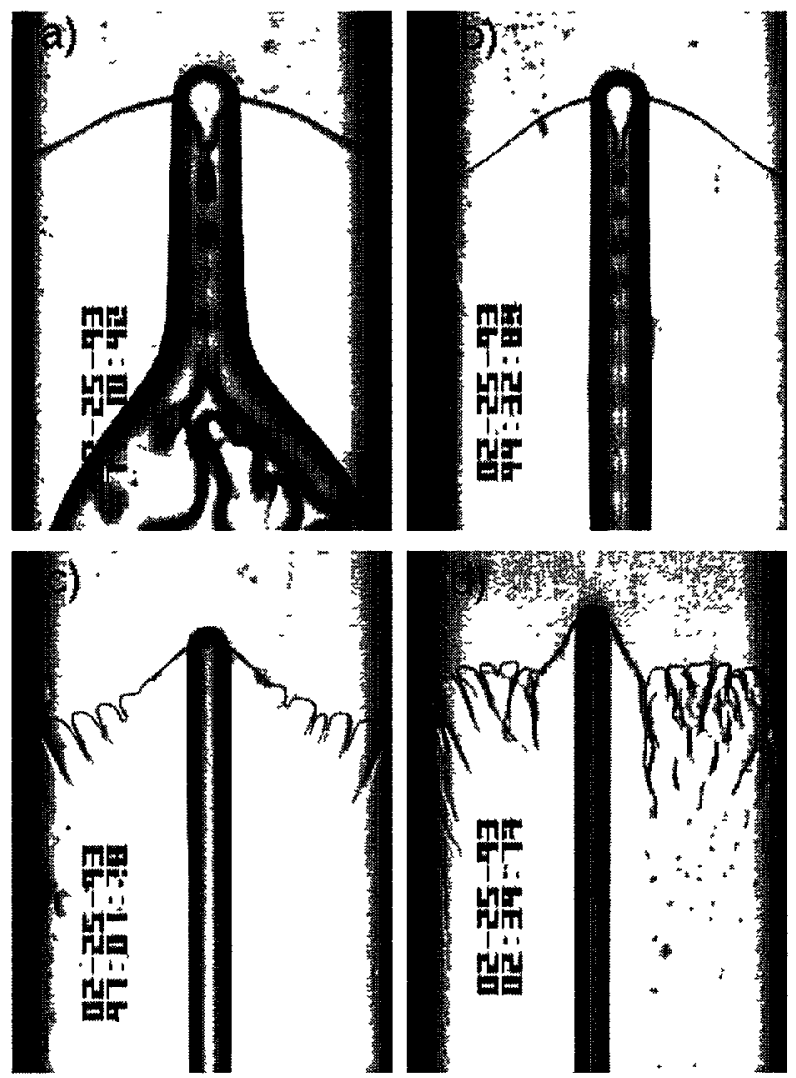

Fig. 15. - A stationary tubular bubble in a doped sample followed through several upward velocity jumps. a) Birth of the tubular bubble at $V=3.8 \mu \mathrm{m} \mathrm{s}^{-1}$, b) $V=4.9 \mu \mathrm{m} \mathrm{s}^{-1}$, c) $V=10.3 \mu \mathrm{m} \mathrm{s}^{-1}$, d) $V=25 \mu \mathrm{m} \mathrm{s}^{-1}$.

visible in Figures $15 \mathrm{a}$ and $15 \mathrm{~b}$, but they clearly correspond to an instability similar to the cusp instability occurring at the groove bottoms of cellular patterns [26], and do not react on the bubble diameter.

The major fact to be noted in Figure 15 is the transition between a low-velocity morphology and a high-velocity one, occurring between about 5 and $10 \mu \mathrm{m} \mathrm{s}^{-1}$ At high velocity, the protruding structure formed by the bubble and the surrounding crystal is obviously local, whereas, at low velocity, the diffusion field attached to the bubble extends over the whole width of the capillary. The term SV finger introduced above appropriately designates the local high-velocity structure (Jamgotchian et al. [9] call it "duplex structure"), while the term tubular regime is more appropriate for the low velocity regime, because of its consonance with the eutectic lamellar regime. Note that, in the low-velocity regime (but not in the high-velocity one), the bubble sits at the centre of the capillary, so that, most probably, the morphology we observe could also exist in the form of an extended periodic pattern of spacing $\lambda$ equal to the width $W_{\mathrm{C}}$ of the capillary $(\approx 450 \mu \mathrm{m})$.

The local character of the SV fingers is established by the following observations:

- the temperature of the cell tips outside the SV finger is unform, and the same as in the absence of bubble; 
- the frontier between the SV finger and the remainder of the front is a sharp one. In the capllary sample, the structure of this frontier is obscured by its $3 \mathrm{D}$ character. In thin samples, the sides of the SV fingers bear sidebranches and end with a deep groove, like those of dendrites;

- the SV fingers have no detectable interactions with distant objects. They can sit steadily at any distance of the container walls. When $W_{\mathrm{F}} \ll W_{\mathrm{C}}$, several SV fingers can coexist within a capillary.

Figure 16 reproduces measured values of the width $W_{\mathrm{B}}$ of the bubble, the width $W_{\mathrm{F}}$ of the SV fingers (taken equal to $W_{\mathrm{C}}$ in the low-velocity regime) as functions of $V$. The shown values correspond to two experiments carried out according to the two above-described procedures. Some quantities measured in undoped samples are also reported. Figure 17 shows the corresponding values of the vapour-phase volume fraction $\eta_{V}$, calculated as the ratio of the transverse cross-section of the bubble (see inset) to $W_{\mathrm{F}} d$, where $d$ is the thickness of the sample. The following points are of particular interest:

1) since, in a stationary state, the average composition of the material left behind by the growth front is $C_{\infty}^{\mathrm{X}}, \eta_{\mathrm{V}}$ should be approximately equal to $C_{\infty}^{\mathrm{X}} v_{\mathrm{V}} / v_{\mathrm{S}}$, where $v_{\mathrm{V}}$ and $v_{\mathrm{S}}$ are the molar volumes of the vapour and the solid. Thus, in a given sample, $\eta_{\mathrm{V}}$ should be independent
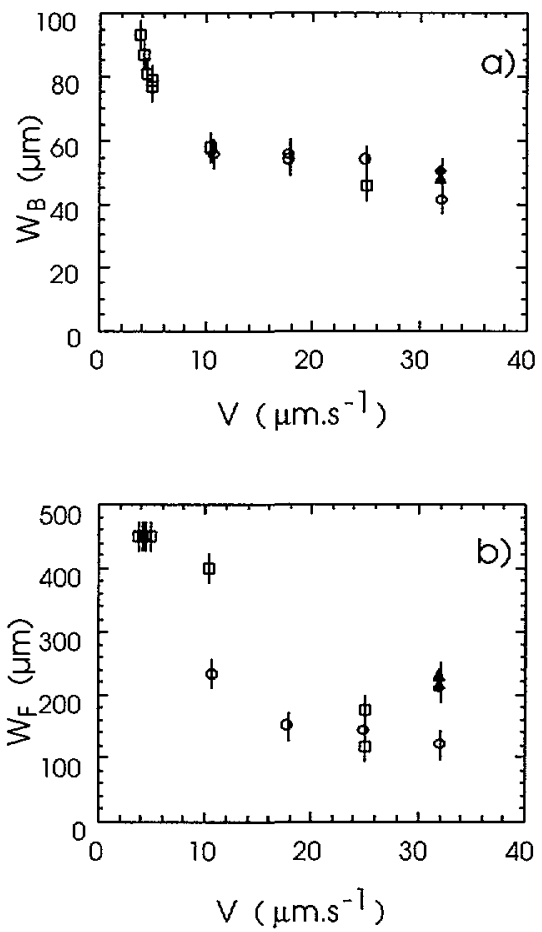

Fig. 16. - a) Measured width $W_{\mathrm{B}}$ of tubular bubbles as a function of the pulling velocity $V$; b) Measured width $W_{F}$ of the corresponding solid-vapour fingers (see inset in Fig. 17). Open symbols: doped samples. Squares: experimental run of Figure 15. Circles: bubble followed through several downward velocity jumps. Closed symbols: undoped samples. Diamonds: capillary sample. Triangles: thin sample. The error bars represent the reading uncertainty. 


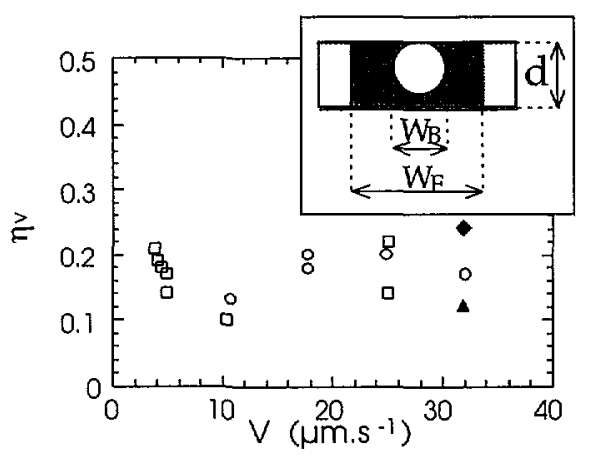

Fig. 17. - Vapour phase fraction $\eta_{\mathrm{V}}$ in the soldd-vapour fingers as a function of the pulling velocity $V$. Inset: sketch of the simplifying assumptions made in the calculation of $\eta_{\mathrm{V}}$. Same symbols as in Figure 16.

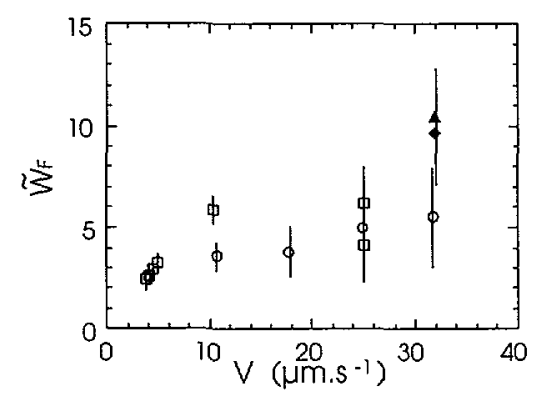

Fig. 18. - Reduced finger width $\widetilde{W}_{\mathrm{F}}=W_{\mathrm{F}} / \ell^{\mathrm{X}}\left(\ell^{\mathrm{x}}\right.$ : diffusion length) as a function of the pulling velocity $V$. Same symbols as in Figure 16.

of $V$. Within experimental uncertainty, this is verified in Figure 17. We find, for all the samples, $\eta_{V}=0.18 \pm 0.08$. This large uncertainty is attributable to the rough assumptions made in the calculation of $\eta_{\mathrm{V}}$. Since $v_{\mathrm{V}} / v_{\mathrm{S}} \approx 250$, this leads us to $C_{\infty}^{\mathrm{X}} \approx 5 \times 10^{-4}$;

2) an important question is whether, in the high-velocity regime, the dimensions of the SV fingers are selected by the system, as are those of the other known local structures, namely, the dendrites, and the recently-discovered symmetry-broken double fingers or "doublons" [14]. In other words, are $W_{\mathrm{B}}$ and $W_{\mathrm{F}}$ well-defined functions of $V$ ? The results presented in Figure 16 are not sufficient in number and accuracy to allow a definitive answer to this question, but they are in favour of the selection hypothesis;

3) this suggests that, in spite of the strong advection-convection flows reigning in the liquid, the shape and the stability of the high-velocity SV finger are essentially diffusion-controlled. If this is true, their width should more or less scale as the diffusion length $\ell^{\mathrm{X}}=D^{\mathrm{X}} / V$. Figure 18 , which gives the values of the reduced width $\widetilde{W}_{\mathrm{F}}=W_{\mathrm{F}} / \ell^{\mathrm{X}}$ supports this assumption. Within experimental uncertainty, $\widetilde{W}_{\mathrm{F}}=4 \pm 1$ in the doped samples and about 10 in the undoped ones (the values of $\widetilde{W}_{\mathrm{B}}$ are irrelevant, since they are largely determined by the large value of $v_{\mathrm{V}} / v_{\mathrm{S}}$ ); 


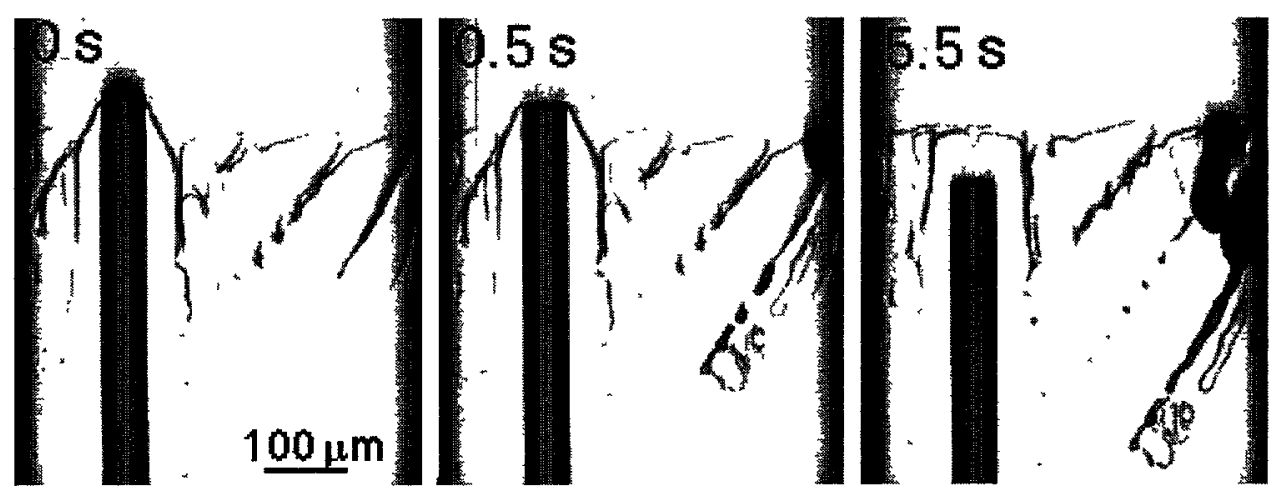

Fig. 19. - A solid-vapour finger killed by the pressure wave generated by the nucleation of another bubble. Doped sample pulled at $V=25 \mu \mathrm{m} \mathrm{s}^{-1}$.

4) at low velocities, the values of $\widetilde{W}_{\mathrm{F}}$ shown in Figure 18 simply represent the Péclet number $\mathrm{Pé}=\lambda / \ell^{\mathrm{X}}$ of the periodic tubular regime, which would exist, presumably, in a sample of large width. Figure 18 shows that this regime is stable for values of Pé larger than unity. This 1s different from the case of eutectic patterns, which, in this velocity range, would only be stable for Pé of the order of $10^{-2}$.

Finally, let us make some remarks about the stability of the tubular-bubble regimes to various types of perturbations. As already mentioned by Jamgotchian et al. [9], it is likely that hydrodynamic couplings occurring via the vortex flows generated in the liquid play an important part in the competition between neighbouring bubbles. This may be the reason for the minimum inter-bubble spacing being large in comparison to the interlamellar spacing of eutectics. In this respect, it is interesting to mention that the bubbles also interact through the pressure waves they generate in the liquid when they change their form. Figure 19 shows that a tubular bubble can be killed by the pressure wave accompanying the emergence of a new bubble into the liquid. Similarly, the bubbles are affected by the pressure changes generated by the transformations occurring in the solid state.

Mechanical couplings apart, tubular bubbles appear quite robust. This is evidenced by the fact that they survive relatively ample velocity jumps. Another aspect of this robustness is the "mobility" of the SV fingers, 1.e., their capability to follow complicated trajectories without being destroyed (see Fig. 13). It has been recently shown that the doublons possess this type of mobility to a high degree because they do not owe their stability to the anisotropy of some property of the interface, and, consequently, do not have a preferential direction of growth [14]. The same reason may also explain the mobility of the SV fingers.

\section{Conclusion}

In this study, our primary purpose was to estimate the perturbations caused by residual impurities on the growth dynamics in directional solidification. From this point of view, our conclusions are as follows. First, the measurement of the initial recoll of the front is a sens1tive, reliable method of testing the quality of the samples. Since it is an in situ method, it reveals the impurities of any origin, including those due to an imperfect cleaning of the exper1mental cells. Its main drawback is that it creates a large macro-segregation in the sample. It is thus generally not possible to test the quality of the material with this method and to perform 
the experiments in the same sample - in other words, the method is, to some extent, destructive. However, in many cases, this drawback can be partly overcome by utilising an alternative method, consisting of following the position (i.e., the temperature) of some particular point of the growth front (cell or dendrite tips, triple points) during the experiment.

A second conclusion is that, quantitatively, the importance of residual-impurity effects is very different according to the aspect of the solidification dynamics considered, and to whether the residual gas remain dissolved or precipitate in the form of bubbles. We have shown that, in spite of the fact that it induces strong convection flows in the liquid, the order of magnitude of the range of action of a stationary tubular bubble is given by the diffusion length. As long as the distance between bubbles remains large compared to this range, there exists, between the bubbles, regions of the front which are hardly affected by their presence. On the other hand, the slow, long-lasting drifts due to the residual impurities remaining in solution are also sources of artefacts. Their most obvious effect is to modify the steady-state value of the undercooling at the growth front $(\Delta T)$, and the value of the cellular-threshold velocity of the system $\left(V_{c}\right)$. More subtle effects are connected with the low value of the partition coefficient of the residual impurities (the observations made by Cladis et al. in succinonitrile [8] show that this is not a circumstance specific of our sole system). As a consequence, both $\Delta T$ and $V_{c}$ increase with time very slowly in comparison with the characteristic duration time of the transients due to the main solute. In practice, this has at least two important consequences: 1 ) the slow increase of $V_{c}$ may give the appearance of being indirect to a cellular bifurcation which is direct; il) for $V>V_{\mathrm{c}}$, the continuous increase of $\Delta T$ is not uniformly distributed along the cellular growth front, but is concentrated in the intercellular grooves. In other words, while the shape of the cell or dendrite tips is not much modified, the temperature and the shape of the front near the bottom of the intercellular grooves vary continuously with time.

As a by-product of this study, we found that the dynamics of solidification fronts in the presence of gas bubbles offers interesting simularities and dissimilarities with the other known types of coupled growth of two phases. At low pulling velocities, it exhibits a stationary permanent state, morphologically analogous to the rod-like pattern of lamellar eutectics and monotectics, but dynamically different in that it is stable at much larger spacings. At higher velocities, it gives rise to a local two-phased structure, the solid-vapour finger, reminiscent of the local structures whose existence has been established, sometimes very recently [27], in cellular and eutectic growth fronts. It will be interesting to go deeper into the dynamic study of these structures in the future.

\section{Acknowledgments}

We are grateful to R. Mellet and A.-M. Pougnet, of the Centre National d'Etudes des Télécommunications, France-Telecom, Bagneux, France, who provided us with zone-refined chemicals. This research was financially supported by the Centre National d'Etudes Spatiales, France.

\section{References}

[1] Wilcox W.R. and Kuo V.H.S., J. Cryst. Growth 19 (1973) 221.

[2] Chen X.-G. and Engler S., Metall 48 (1994) 276.

[3] Kutumbarao V.V. and Greenwood G.W., Mater. Sci. Technol. 2 (1994) 129. 
[4] Chernov A.A. and Temkin D.E., in "Crystal Growth and Materials, E. Kaldis and H.-J. Scheel Eds. (North-Holland, Amsterdam, 1977) p. 1.

[5] Gegusin J.E. and Dzjuba A.S., Krystallografiya 22 (1977) 348.

[6] Laherrère J.-M., Mellet R., Savary H., Licoppe C., Scott J.F. and Tolédano J.-C., Phys. Rev. A 42 (1990) 3634; Laherrère J.-M., Tolédano J.-C., Savary H. and Mellet R., Europhys. Lett. 15 (1991) 197.

[7] Williams L.M., Srinavasan M.R. and Cummins H.Z., Phys. Rev. Lett. 64 (1990) 1526.

[8] Cladis P.E., Gleeson J.T. and Finn P.L., in "Patterns and Instabilities", D. Walgraef and N. Ghoniem Eds. (Kluwer, Dordrecht, 1990).

[9] Jamgotchian H., Trivedi R. and Billia B., J. Cryst. Growth 134 (1993) 181.

[10] Mergy J., Faivre G., Guthmann C. and Mellet R., J. Cryst. Growth 134 (1993) 353. A fortuitous error made us write $m^{\mathrm{H}} \approx 81{ }^{\circ} \mathrm{C}$ for the average value of the liquidus slope on the $\mathrm{CBr}_{4}$ side (p. 357). In fact, $81^{\circ} \mathrm{C}$ is the maximum value of $m^{\mathrm{H}}$, occurring at the eutectic concentration, the average value being $70^{\circ} \mathrm{C}$. The difference between the two values is however hardly larger than the sum of experimental uncertainties on each value.

[11] Battino R. and Clever H.L., Chem. Rev. 66 (1977) 395.

[12] Jackson K.A. and Hunt J.D., Acta Metall. 13 (1965) 1212.

[13] Faivre G. and Mergy J., Phys. Rev. A 45 (1992) 7320; 46 (1992) 963.

[14] Thle T. and Müller-Krumbhaar H., Phys. Rev. Lett. 70 (1993) 3083; Phys. Rev. E 49 (1994) 2972; Akamatsu S., Faivre G. and Ihle T., Phys. Rev. E 51 (1995) 4751.

[15] Smith V.G., Tiller W.A. and Rutter J.W., Can. J. Phys. 33 (1955) 723.

[16] Jackson K.A. and Hunt J.D., Trans. AIME 236 (1966) 1129.

[17] Grugel R.N. and Hellawell A., Metall. Trans. 13A (1982) 493.

[18] Lebrun M.-M., Thèse de Doctorat, Univ. Parıs 7 (1987); de Chevelgné S., Guthmann C. and Lebrun M.-M., J. Phys. France 47 (1986) 2095.

[19] More M., Lefèbvre J. and Fouret R., Acta Cryst. B 333681 and 3862 (1977).

[20] Mc Call D.W., Douglass D.C. and Anderson E.W., J. Chem. Phys 31 (1959) 1555.

[21] Tiller W.A., J. Appl. Phys. 34 (1963) 2757.

[22] Warren J.A. and Langer J.S., Phys. Rev. A 47 (1993) 2702. The WL approximation does not lead to explicit formulae, but replaces the equations of growth with two coupled ordinary differential equations.

[23] Caroli B., Caroli C. and Ramirez-Piscina L., J. Cryst. Growth 132 (1993) 377.

[24] Measuring the cellular threshold velocity involves a careful discussion of the 3D-effects, 1.e., the deformations of the growth front in the direction perpendicular to the plane of the sample. We hope to report on the 3D effects in thin-film directional solidification in a future article.

[25] Mergy J., Thèse de Doctorat, Univ. Paris 7 (1992).

[26] Kurowski P., de Chevelgné S., Faivre G. and Guthmann C., J. Phys. France 50 (1989) 3007.

[27] A two-phase local structure, named $\alpha$ - $\beta$ finger, has recently been identified in lamellar eutectic growth front. Morphologically, it is quite similar to the SV finger, since it is constituted of a thin lamella of one of the two eutectic crystal phases surrounded by a sheath of the other crystal phase. Dynamically, its local character and the degeneracy of its growth direction are very pronounced. Its appearance perhaps depends on the presence of the third (residual) component in the alloy (Ginibre M., Thèse de Doctorat, Université Pierre-et-Marie Curie, in preparation). 